\title{
Flow of a viscous compressible fluid produced in a circular tube by an impulsive point source
}

\author{
B. U. FELDERHOF ${ }^{1}$ AND G. OOMS ${ }^{2} \dagger$ \\ ${ }^{1}$ Institut für Theoretische Physik A, RWTH Aachen University, Templergraben 55, \\ 52056 Aachen, Germany \\ ${ }^{2}$ J. M. Burgerscentrum, Laboratory for Aero and Hydrodynamics, \\ Delft University of Technology, Mekelweg 2, 2628 CD Delft, The Netherlands
}

(Received 16 July 2010; revised 1 September 2010; accepted 15 October 2010)

The flow of a viscous compressible fluid in a circular tube generated by a sudden impulse at a point on the axis is studied on the basis of the linearized Navier-Stokes equations. A no-slip boundary condition is assumed to hold on the wall of the tube. An efficient numerical scheme has been developed for the calculation of flow velocity and pressure disturbance as a function of position and time.

Key words: compressible flows

\section{Introduction}

Compressibility has a significant effect on the dynamics of the flow of a viscous fluid in a circular tube. Because of the finite velocity of sound, the local disturbance of a quiescent fluid generated by a sudden impulse at a chosen point occurs only after a finite travelling time, rather than instantaneously, as in an incompressible fluid. As pointed out by Pagonabarraga et al. (1999), confinement by rigid boundaries strongly affects the sound modes in a compressible fluid. In earlier work by one of us (Felderhof 2010a), analytic expressions were derived for flow velocity and pressure in terms of Fourier integrals for a source point chosen on the axis. In particular, the wave reflected by the tube requires the evaluation of a double Fourier integral over wavenumber and frequency. Although numerical results were presented for the flow and pressure at selected points, a complete picture of flow velocity and pressure as functions of position and time was still missing. We have constructed a scheme of numerical evaluation based on the technique of fast Fourier transform (Brigham 1974). The scheme allows fast calculation of flow velocity and pressure.

The analysis is based on the solution of the linearized Navier-Stokes equations for a compressible viscous fluid. We consider in particular a sudden point impulse on the axis of the tube and in the direction of the axis. The case of an impulse perpendicular to the axis can be studied by the same technique (Felderhof 2010b). In the recent work of Frydel \& Diamant (2010), the Green function for the parallel case is used to discuss a transient hydrodynamic pair interaction between suspended particles centred on the axis of the tube.

The numerical results for flow velocity and pressure depend strongly on a dimensionless number characterizing the system. For a tube of radius $b$ the viscous 
relaxation time $\tau_{v}$ may be defined as $\tau_{v}=b^{2} / \nu$, where $v$ is the kinematic viscosity. The characteristic time for sound propagation is $\tau_{s}=b / c_{0}$, where $c_{0}$ is the longwavelength sound velocity. The ratio of the two times is the dimensionless number $R_{b}=b c_{0} / v$. This may be expressed alternatively as the ratio of the Reynolds number $R e=U b / v$ to Mach number $M a=U / c_{0}$, where $U$ is a characteristic velocity. In the ratio $R_{b}=R e / M a$, the velocity $U$ cancels. On account of the linearization of the Navier-Stokes equations, the Reynolds number does not play a separate role, and the results apply only to a weak impulse. As a reference, for water $v=0.01 \mathrm{~cm}^{2} \mathrm{~s}^{-1}$ and with $c_{0}=1500 \mathrm{~m} \mathrm{~s}^{-1}$ and a tube radius $b=10^{-4} \mathrm{~cm}$, it is found that $R_{b}=1500$.

We shall consider the two values $R_{b}=100$, corresponding to a nearly incompressible fluid, and $R_{b}=5.3$, corresponding to the numerical simulation of Hagen et al. (1997) and Pagonabarraga et al. (1999). In the recent simulation of Frydel \& Diamant (2010), the value is $R_{b}=26$. For $R_{b}=100$ (or $R_{b}=1500$ ), the viscous relaxation time is much larger than the characteristic time for sound propagation and the influence of viscous effects on the initial wave propagation is rather small. However, at a value of $R_{b}=5.3$, the influence of viscosity on, for instance, the broadening of the acoustic wavefront is significant. For a given fluid, the number $R_{b}$ is small in nanogeometry, and it increases in proportion to the width of the tube. It is of interest to study the dynamics of the flow in a wide range of circumstances.

\section{Linear hydrodynamics of the flow in a circular tube}

We consider a viscous compressible fluid with shear viscosity $\eta$, bulk viscosity $\eta_{v}$ and equilibrium mass density $\rho_{0}$ located in a circular tube of radius $b$. We choose coordinates such that the $z$-axis is along the axis of the tube and we use cylindrical coordinates $(R, \varphi, z)$. For time $t<0$, the fluid is at rest with static pressure $p_{s}$. At time $t=0$, an impulse $\boldsymbol{P}$ is imparted to the fluid at the origin and directed along the $z$-axis. We study the resulting motion of the fluid for time $t>0$. In this way, the Green function of the flow problem is determined. This function is the building block for more complex acoustic sources.

For small-amplitude motion, the flow velocity $\boldsymbol{v}(\boldsymbol{r}, t)$ and the pressure $p(\boldsymbol{r}, t)$ are governed by the linearized Navier-Stokes equations and continuity equation:

$$
\left.\begin{array}{rl}
\rho_{0} \frac{\partial \boldsymbol{v}}{\partial t} & =\eta \nabla^{2} \boldsymbol{v}+\left(\frac{1}{3} \eta+\eta_{v}\right) \nabla \nabla \cdot \boldsymbol{v}-\nabla p+\boldsymbol{P} \delta(\boldsymbol{r}) \delta(t), \\
\frac{\partial p}{\partial t} & =-\rho_{0} c_{0}^{2} \nabla \cdot \boldsymbol{v},
\end{array}\right\}
$$

with impulse $\boldsymbol{P}=P \boldsymbol{e}_{z}$ and long-wave sound velocity $c_{0}$. We assume that the flow velocity satisfies the no-slip boundary condition at the wall of the cylinder, i.e. $\boldsymbol{v}=\mathbf{0}$ at $R=b$. We look for the solution of (2.1) for which the flow velocity $\boldsymbol{v}(\boldsymbol{r}, t)$ vanishes and the pressure tends to the static pressure $p_{s}$, as $z \rightarrow \pm \infty$ at any time $t$.

After Fourier analysis in time, we find that the equations for the Fourier components

$$
\boldsymbol{v}_{\omega}(\boldsymbol{r})=\int_{0}^{\infty} \mathrm{e}^{\mathrm{i} \omega t} \boldsymbol{v}(\boldsymbol{r}, t) \mathrm{d} t, \quad p_{\omega}(\boldsymbol{r})=\int_{0}^{\infty} \mathrm{e}^{\mathrm{i} \omega t}\left[p(\boldsymbol{r}, t)-p_{s}\right] \mathrm{d} t
$$

are

$$
\begin{aligned}
\eta\left(\nabla^{2} \boldsymbol{v}_{\omega}-\alpha^{2} \boldsymbol{v}_{\omega}\right)+\left(\frac{1}{3} \eta+\eta_{v}\right) \nabla \nabla \cdot \boldsymbol{v}_{\omega}-\nabla p_{\omega} & =-\boldsymbol{P} \delta(\boldsymbol{r}), \\
\nabla \cdot \boldsymbol{v}_{\omega}-\mathrm{i} \beta p_{\omega} & =0
\end{aligned}
$$


where we have used the abbreviations

$$
\alpha=\sqrt{\frac{-\mathrm{i} \omega \rho_{0}}{\eta}}, \quad \operatorname{Re} \alpha>0, \quad \beta=\frac{\omega}{\rho_{0} c_{0}^{2}} .
$$

We write the Fourier-transformed flow velocity as

$$
\boldsymbol{v}_{\omega}(\boldsymbol{r})=\boldsymbol{v}_{0 \omega}(\boldsymbol{r})+\boldsymbol{v}_{1 \omega}(\boldsymbol{r}),
$$

where $\boldsymbol{v}_{0 \omega}(\boldsymbol{r})$ is the solution for infinite space and $\boldsymbol{v}_{1 \omega}(\boldsymbol{r})$ is the reflected flow due to the presence of the boundary. The flows can be expressed as

$$
\boldsymbol{v}_{0 \omega}(\boldsymbol{r})=\boldsymbol{G}_{0}\left(\boldsymbol{r}-\boldsymbol{r}_{0}\right) \cdot \boldsymbol{P}, \quad \boldsymbol{v}_{\omega}(\boldsymbol{r})=\boldsymbol{G}\left(\boldsymbol{r}, \boldsymbol{r}_{0}\right) \cdot \boldsymbol{P},
$$

with Green functions $\boldsymbol{G}_{0}$ and $\boldsymbol{G}$. The Green function for infinite space is translationally invariant and given explicitly by (Jones 1981)

$$
\boldsymbol{G}_{0}(\boldsymbol{r})=\frac{1}{4 \pi \eta}\left(\frac{\mathrm{e}^{-\alpha r}}{r} \mathbf{1}+\alpha^{-2} \nabla \nabla \frac{\mathrm{e}^{\mathrm{i} / \mu r}-\mathrm{e}^{-\alpha r}}{r}\right),
$$

with the abbreviation

$$
\mu=\omega / c, \quad \operatorname{Im} \mu>0,
$$

where

$$
c=c_{0} \sqrt{1-\mathrm{i} \omega \tau_{p}} \quad \tau_{p}=\frac{4 \eta+3 \eta_{v}}{3 \rho_{0} c_{0}^{2}} .
$$

The corresponding expression for the pressure in infinite space is

$$
p_{0 \omega}(\boldsymbol{r})=\frac{P}{4 \pi} \frac{c_{0}^{2}}{c^{2}} \frac{z}{r^{3}}(1-\mathrm{i} \mu r) \mathrm{e}^{\mathrm{i} \mu r} .
$$

The reflected flow velocity $\boldsymbol{v}_{1 \omega}$ is expressed as (Felderhof 2010a)

$$
\left.\begin{array}{rl}
v_{1 R \omega}(\boldsymbol{r}) & =\frac{P}{2 \pi^{2} \eta \alpha^{2}} \int_{0}^{\infty} \hat{v}_{1 R}(k, \omega, R) \sin k z \mathrm{~d} k, \\
v_{1 z \omega}(\boldsymbol{r}) & =\frac{P}{2 \pi^{2} \eta \alpha^{2}} \int_{0}^{\infty} \hat{v}_{1 z}(k, \omega, R) \cos k z \mathrm{~d} k,
\end{array}\right\}
$$

with amplitudes

$$
\left.\begin{array}{rl}
\hat{v}_{1 R}(k, \omega, R) & =A(k, \omega) \hat{v}_{R p}(k, \omega, R)+B(k, \omega) \hat{v}_{R v}(k, \omega, R), \\
\hat{v}_{1 z}(k, \omega, R) & =A(k, \omega) \hat{v}_{z p}(k, \omega, R)+B(k, \omega) \hat{v}_{z v}(k, \omega, R),
\end{array}\right\}
$$

where

$$
\begin{gathered}
\hat{v}_{R p}(k, \omega, R)=k u \mathrm{I}_{1}(u R), \quad \hat{v}_{R v}(k, \omega, R)=k s \mathrm{I}_{1}(s R), \\
\hat{v}_{z p}(k, \omega, R)=k^{2} \mathrm{I}_{0}(u R), \quad \hat{v}_{z v}(k, \omega, R)=s^{2} \mathrm{I}_{0}(s R),
\end{gathered}
$$

with modified Bessel functions $\mathrm{I}_{n}(x)$ and the abbreviations

$$
s=\sqrt{k^{2}+\alpha^{2}}, \quad u=\sqrt{k^{2}-\mu^{2}} .
$$

The coefficients $A(k, \omega)$ and $B(k, \omega)$ are given by

$$
A(k, \omega)=\frac{P(k, \omega)}{Z(k, \omega)}, \quad B(k, \omega)=\frac{Q(k, \omega)}{Z(k, \omega)},
$$


with denominator

$$
Z(k, \omega)=b s\left[u s \mathrm{I}_{0}(s b) \mathrm{I}_{1}(u b)-k^{2} \mathrm{I}_{0}(u b) \mathrm{I}_{1}(s b)\right]
$$

and numerators

$$
\left.\begin{array}{l}
P(k, \omega)=s^{2}-s k^{2} b \mathrm{~K}_{0}(u b) \mathrm{I}_{1}(s b)-s^{2} u b \mathrm{I}_{0}(s b) \mathrm{K}_{1}(u b), \\
Q(k, \omega)=k^{2}-s^{2} u b \mathrm{~K}_{0}(s b) \mathrm{I}_{1}(u b)-k^{2} s b \mathrm{I}_{0}(u b) \mathrm{K}_{1}(s b),
\end{array}\right\}
$$

with modified Bessel functions $\mathrm{K}_{n}(x)$. The corresponding expression for the pressure is

$$
p_{1 \omega}(\boldsymbol{r})=\frac{P}{2 \pi^{2}} \frac{c_{0}^{2}}{c^{2}} \int_{0}^{\infty} A(k, \omega) \mathrm{I}_{0}(u R) k \sin k z \mathrm{~d} k .
$$

We note that the flow components $v_{0 R}(R, z, t)$ and $v_{1 R}(R, z, t)$ and the pressure contributions $p_{0}(R, z, t)$ and $p_{1}(R, z, t)$ are odd functions of $z$, whereas the flow components $v_{0 z}(R, z, t)$ and $v_{1 z}(R, z, t)$ are even functions of $z$.

In order to evaluate the flow fields $\boldsymbol{v}_{0}(\boldsymbol{r}, t)$ and $\boldsymbol{v}_{1}(\boldsymbol{r}, t)$ and the corresponding pressures $p_{0}(\boldsymbol{r}, t)$ and $p_{1}(\boldsymbol{r}, t)$, the Fourier transforms must be inverted numerically. Only the viscous part of the flow field $\boldsymbol{v}_{0}(\boldsymbol{r}, t)$ can be found in an analytical form. Writing

$$
\boldsymbol{v}_{0}(\boldsymbol{r}, t)=\boldsymbol{v}_{0 v}(\boldsymbol{r}, t)+\boldsymbol{v}_{0 p}(\boldsymbol{r}, t)
$$

one has for the components in spherical coordinates $(r, \theta, \varphi)$ of the viscous part (Cichocki \& Felderhof 2000)

$$
\left.\begin{array}{l}
v_{0 v r}(\boldsymbol{r}, t)=\frac{P}{4 \pi \eta}\left[-\frac{2 v}{r^{3}} \operatorname{erfc} \frac{r}{\sqrt{4 v t}}-\frac{2}{r^{2}} \sqrt{\frac{v}{\pi t}} \mathrm{e}^{-r^{2} / 4 v t}\right] \cos \theta, \\
v_{0 v \theta}(\boldsymbol{r}, t)=\frac{P}{4 \pi \eta}\left[-\frac{v}{r^{3}} \operatorname{erfc} \frac{r}{\sqrt{4 v t}}-\frac{r^{2}+2 v t}{2 \sqrt{\pi v} r^{2} t^{3 / 2}} \mathrm{e}^{-r^{2} / 4 v t}\right] \sin \theta,
\end{array}\right\}
$$

where $v=\eta / \rho_{0}$ is the kinematic viscosity and $\cos \theta=z / r$. The non-vanishing Fourier components of the pressure part of the flow are

$$
\left.\begin{array}{l}
v_{0 p \omega r}(\boldsymbol{r})=\frac{P}{4 \pi \eta \alpha^{2} r^{3}}\left(2-2 \mathrm{i} \mu r-\mu^{2} r^{2}\right) \mathrm{e}^{\mathrm{i} \mu r} \cos \theta \\
v_{0 p \omega \theta}(\boldsymbol{r})=\frac{P}{4 \pi \eta \alpha^{2} r^{3}}(1-\mathrm{i} \mu r) \mathrm{e}^{\mathrm{i} \mu r} \sin \theta .
\end{array}\right\}
$$

The flow field $\boldsymbol{v}_{0 p}(\boldsymbol{r}, t)$ and the pressure $p_{0}(\boldsymbol{r}, t)$ can be found by numerical Fourier inversion with respect to frequency $\omega$ only of the expressions in (2.10) and (2.21). In that case, the inverse Fourier transform is only one-dimensional and can be performed without difficulty. Only this part of the flow is singular. The numerical calculation of the reflected flow field $\boldsymbol{v}_{1}(\boldsymbol{r}, t)$ and of the corresponding pressure $p_{1}(\boldsymbol{r}, t)$ requires numerical inversion of the Fourier transforms in (2.11) and (2.18) in both wavenumber $k$ and frequency $\omega$. The latter calculation can be performed efficiently with the aid of the fast Fourier transform technique (Brigham 1974). The range of frequencies used is of the order of 100 and the number of frequencies in that range is of the order of 200. This holds also for the wavenumber. For moderate values of $r$ and $t$, it is also possible to calculate the reflected flow field and the corresponding pressure by direct integration of the Fourier integrals with respect to frequency and wavenumber. The results agree well with those derived by using the fast Fourier transform technique. Such direct calculation requires significantly more computing time. 


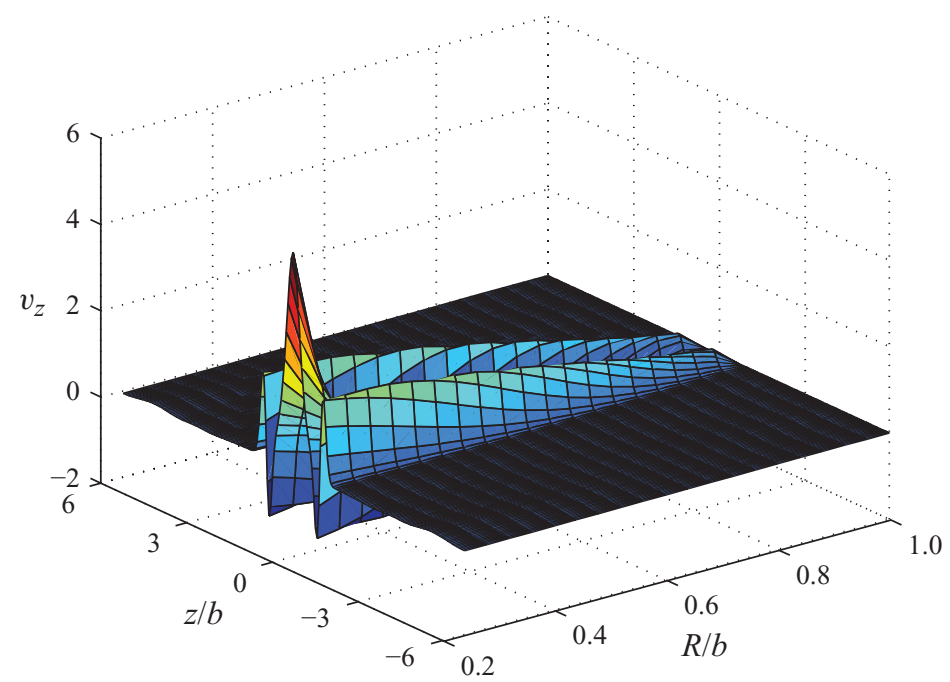

FIGURE 1. Plot of the axial velocity $v_{z}$ as a function of the axial coordinate $z / b$ and the radial coordinate $R / b$ at time $t=1$ and for $\eta=0.01$ and $\eta_{v}=0$. In order to see the details of the pressure waves, only the velocity field for values of $R / b>0.2$ is shown. In that region, the value of the velocity peak is already lower. The same region will be shown in all following figures of the velocity and pressure field.

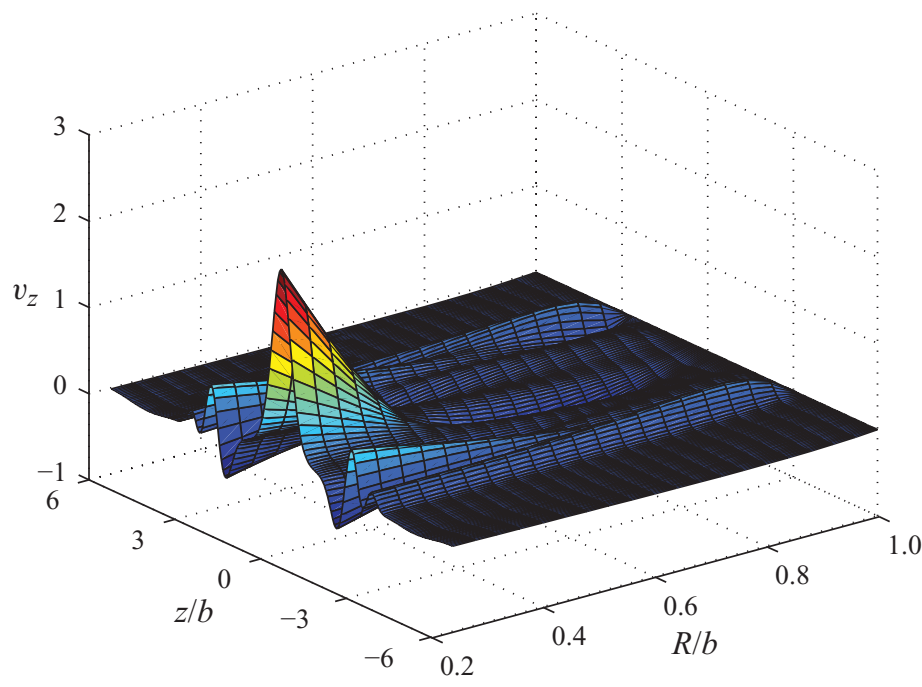

FIGURE 2. Plot of the axial velocity $v_{z}$ as a function of the axial coordinate $z / b$ and the radial coordinate $R / b$ at time $t=3$ and for $\eta=0.01$ and $\eta_{v}=0$.

\section{Flow velocity and pressure}

We choose the unit of length such that $b=1$, the unit of time such that $c_{0}=1$, and the unit of mass such that $\rho_{0}=1$. Then, for shear viscosity $\eta=0.01$, the dimensionless number is $R_{b}=100$. We choose $\eta_{v}=0$, corresponding to a dilute gas. The sound velocity $c_{0}$ is large compared with the viscous velocity $v / b$. In figures 1,2 , and 3 , we show the axial velocity component $v_{z}(\boldsymbol{r}, t)$ as a function of $z / b$ and $R / b$ at the three subsequent times $t=1,3$ and 5 , respectively. To avoid the strong peak near the 


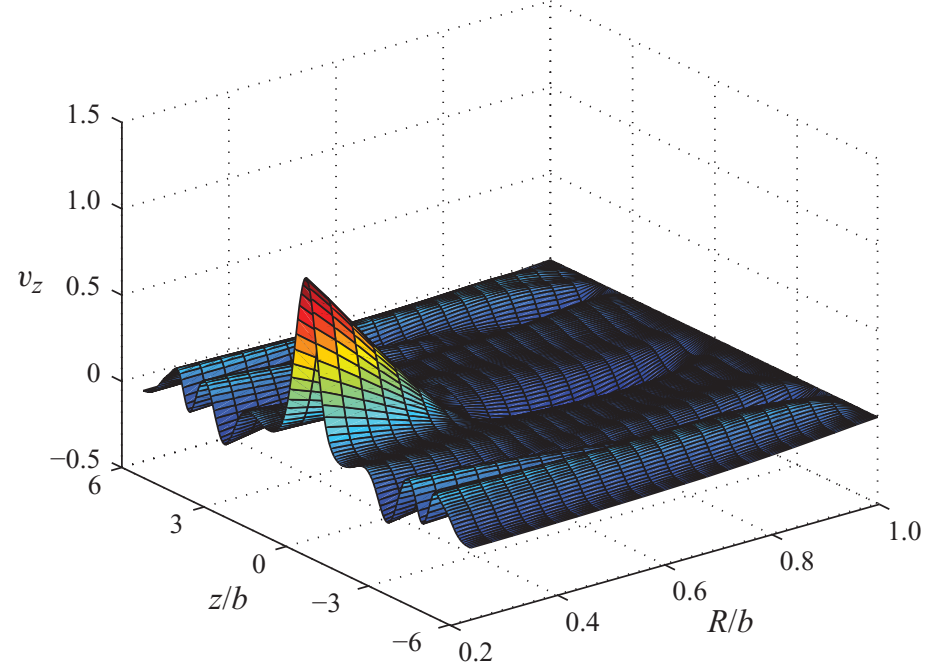

FIGURE 3. Plot of the axial velocity $v_{z}$ as a function of the axial coordinate $z / b$ and the radial coordinate $R / b$ at time $t=5$ and for $\eta=0.01$ and $\eta_{v}=0$.

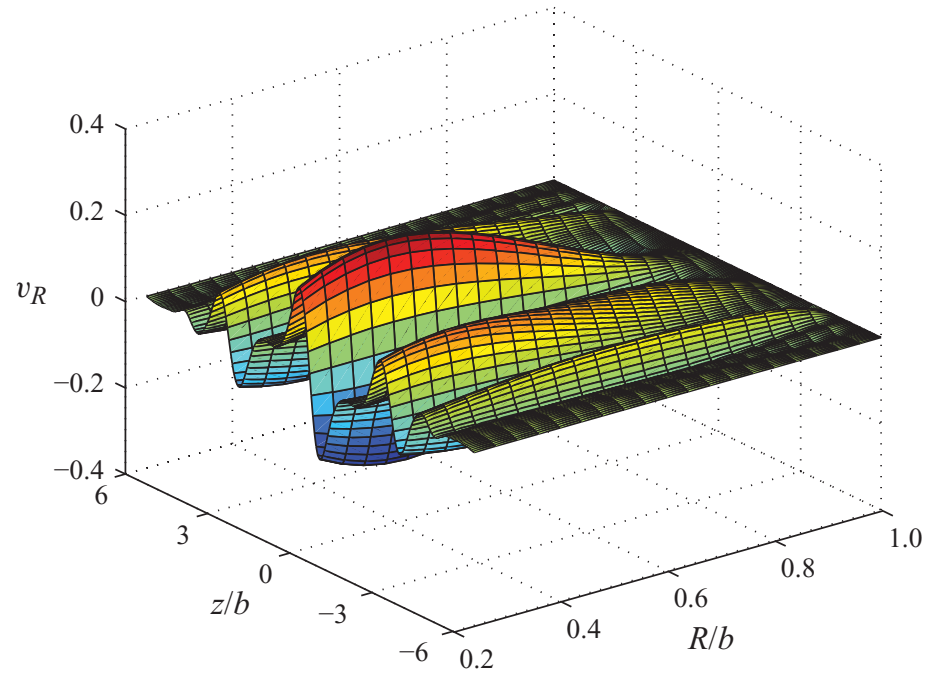

FIGURE 4. Plot of the radial velocity $v_{R}$ as a function of the axial coordinate $z / b$ and the radial coordinate $R / b$ at time $t=5$ and for $\eta=0.01$ and $\eta_{v}=0$.

origin, we plot only the region $R / b>0.2 b$. In figure 4 , we show the radial velocity component $v_{R}(\boldsymbol{r}, t)$ as a function of $z / b$ and $R / b$ at time $t=5$. For small axial distance, there is a strong axially symmetric vortex ring. Owing to the point source at $(z / b=0, R / b=0)$, there exists a strong flow in the positive axial direction along the centreline of the tube $(R / b=0)$. By symmetry, it suffices to consider the flow in a single azimuthal plane. At a small axial distance, the flow starts to bend in the positive radial direction, then flows in the negative axial direction along the tube wall and finally bends back in the negative radial direction, thus completing the cycle. In figure 5, we show the streamlines at time $t=2$ in the plane $\varphi=0$. Pagonabarraga et al. (1999) studied both the geometry of a tube and of a fluid between parallel plane 


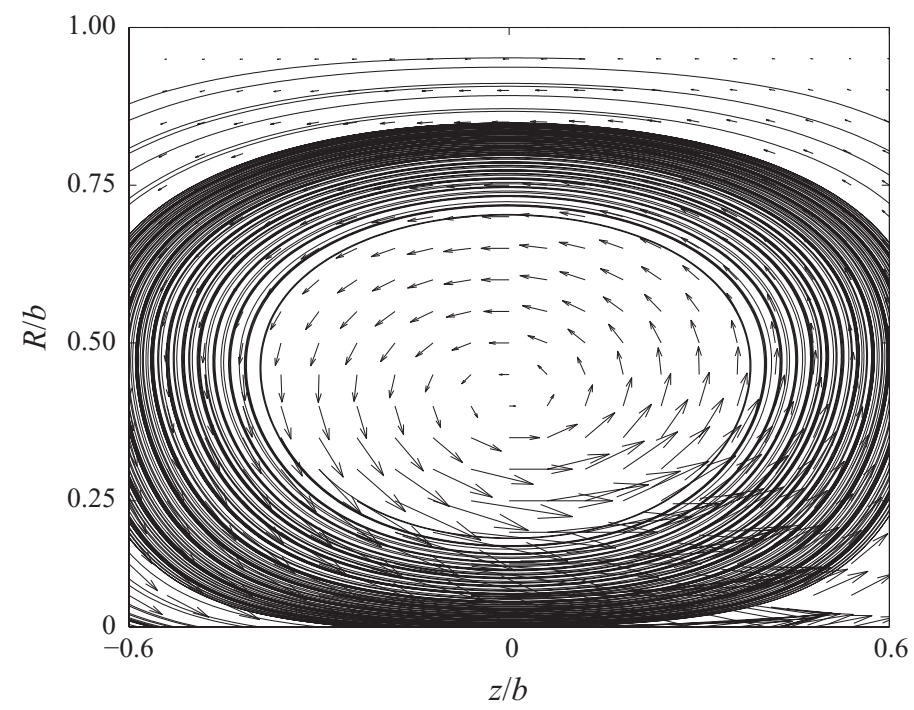

FIGURE 5. Plot of the velocity vectors and streamlines in the vortex ring at $t=2$ and for $\eta=0.01$ and $\eta_{v}=0$.

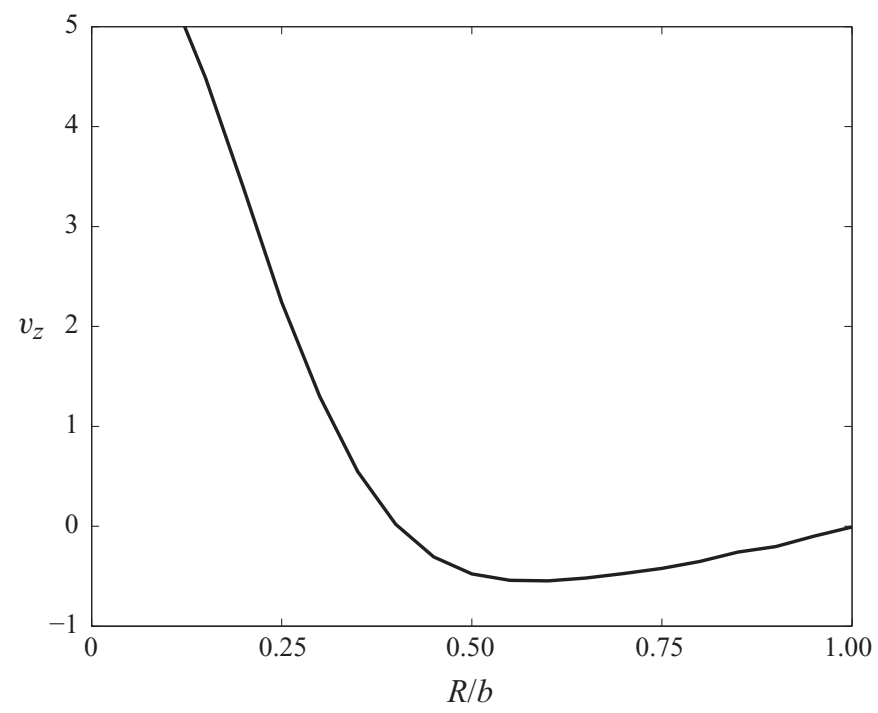

FIGURE 6. Plot of the axial velocity $v_{z}$ as a function of the radial coordinate $R / b$ at $z / b=0$ and $t=2$ and for $\eta=0.01$ and $\eta_{v}=0$.

walls. In their plane wall studies, they found that there are subsidiary vortices set up and that the primary vortices decay very rapidly. The work of Liron \& Shahar (1978) shows that in the steady-state limit, which corresponds to the integral over time of the transient flow, there is an infinite sequence of eddies along the axis of the tube. For the range of time covered by our study, we see no subsidiary vortices beyond three diameters upstream or downstream of the point source. The primary vortex decays indeed rather rapidly: as can be seen from figures $1-3$, the amplitude decays a factor of 5 between $t=1$ and $t=5$. In figure 6 , we show the axial velocity component in the plane $z=0$ at time $t=2$ as a function of radial distance $R / b$. There is a strong flow 


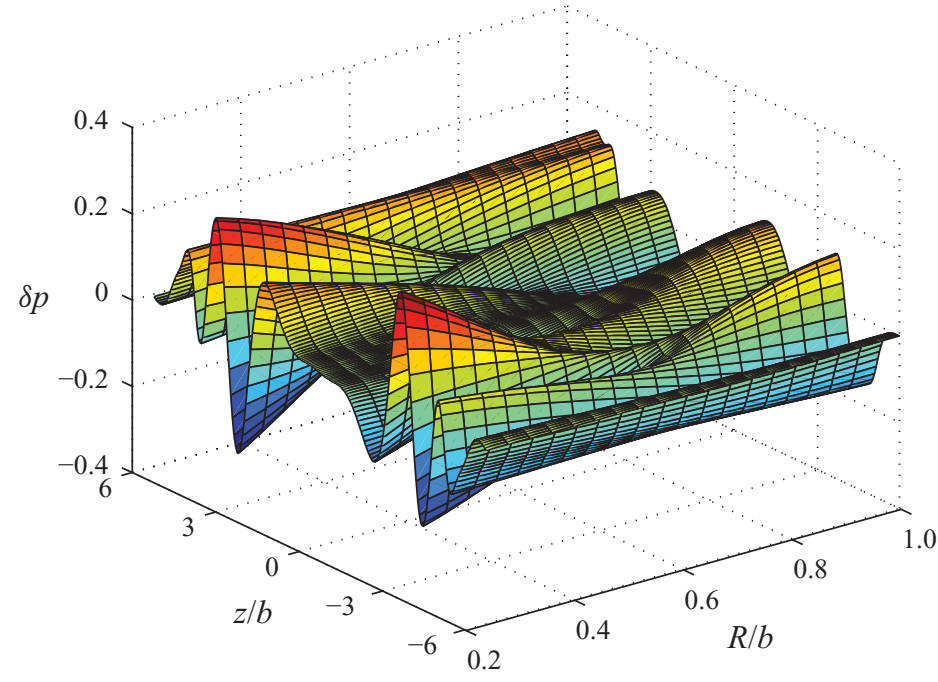

FIgURE 7. Plot of the pressure $\delta p$ as a function of the axial coordinate $z / b$ and the radial coordinate $R / b$ at time $t=5$ and for $\eta=0.01$ and $\eta_{v}=0$.

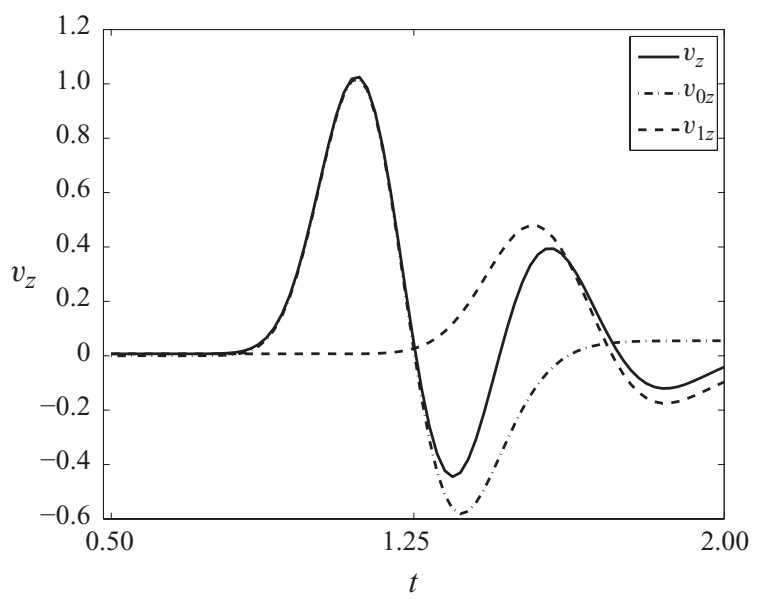

FIGURE 8. Plot of the axial velocity $v_{z}$ at the selected point $(z / b=1, R / b=0.7)$ as a function of time $t$, as well as the separate contributions from the outgoing wave $v_{0 z}$ and the reflected wave $v_{1 z}$ for $\eta=0.01$ and $\eta_{v}=0$.

in the positive $z$-direction for small radial distances, compensated by a backflow for larger radial distances. For larger values of $|z / b|$, the flow is dominated by pressure waves. In figure 7, we show the pressure disturbance $\delta p(\boldsymbol{r}, t)$ as a function of $z / b$ and $R / b$ at time $t=5$. The total pressure is $p(\boldsymbol{r}, t)=p_{s}+\delta p(\boldsymbol{r}, t)$. In figure 8 , we show the axial velocity component $v_{z}$ at the selected point $R / b=0.7, \varphi=0, z / b=1$ as a function of time, as well as the separate contributions from the outgoing wave $v_{0 z}$ and the reflected wave $v_{1 z}$. An additional calculation with $\eta_{v}=0.1$ (keeping all other parameters the same) was made. There are no noticeable changes with respect to the $\eta_{v}=0$ case. A more systematic study is needed to investigate the influence of $\eta_{v}$ in detail. 


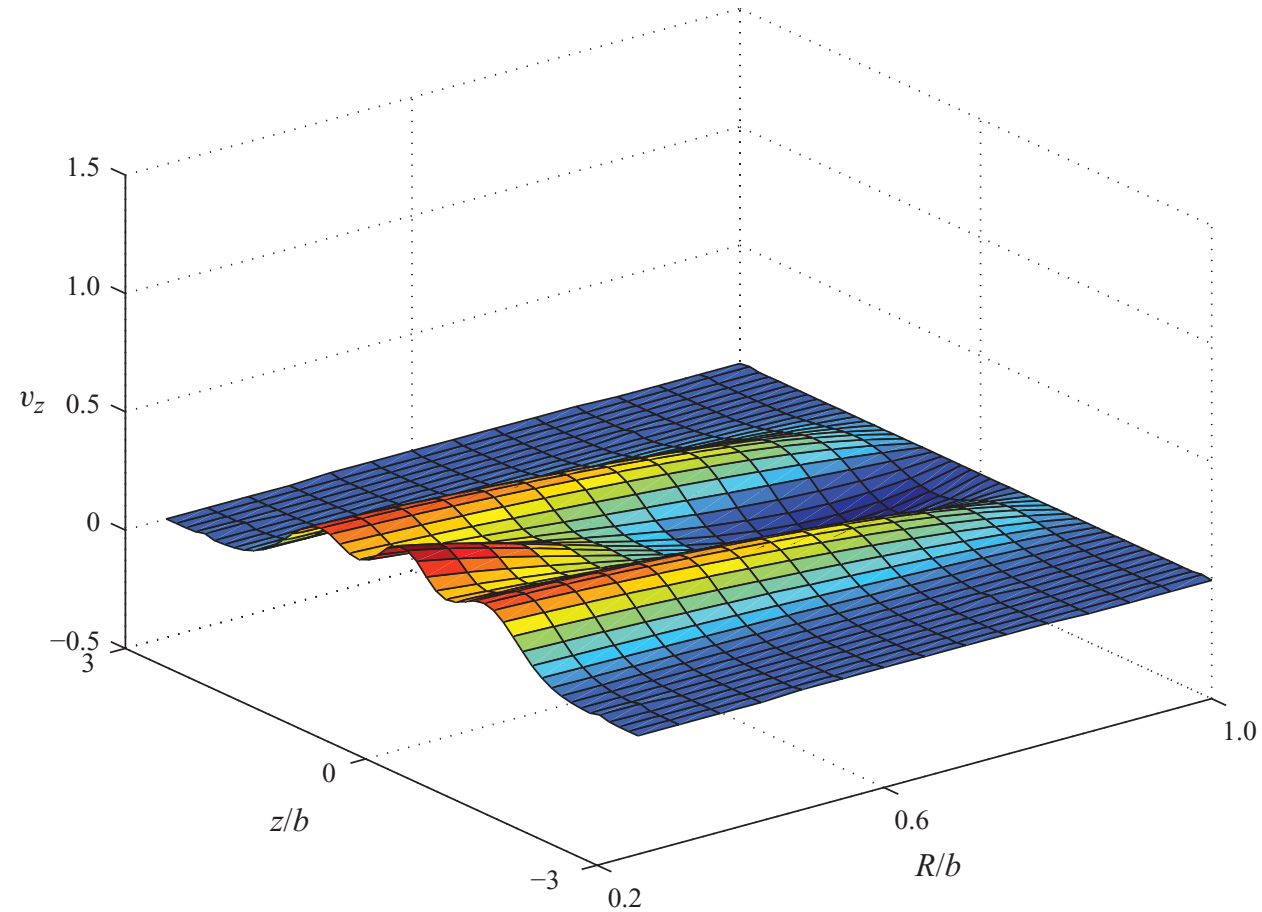

FIGURE 9. Plot of the axial velocity $v_{z}$ as a function of the axial coordinate $z / b$ and the radial coordinate $R / b$ at time $t=1$ and for $\eta=0.133$ and $\eta_{v}=0.00044$. A comparison with figure 1 shows the influence of the increase in viscosity on the velocity field. Like in figure 1, only the velocity field for values of $R / b>0.2$ is shown. It is evident from the comparison that the viscosity has a very strong damping effect. In order to see the development in time towards $t=1$, in figures 10 and 11 , we show the flow field at $t=0.4$ and $t=0.7$, respectively.

For comparison, we show corresponding figures for the fluid from the computer simulations of Hagen et al. (1997) and Pagonabarraga et al. (1999). We use the same units of length, time and mass as before, such that $b=1, c_{0}=1$ and $\rho_{0}=1$. Then, $\eta=1 / 7.5$ yields the value $R_{b}=5.3$ used in the computer simulation. We use the corresponding value $\eta_{v}=0.00044$ for the volume viscosity (D. Frenkel \& Lowe 2005, personal communication). In effect, the fluid is 13.3 times more viscous than in the previous case, corresponding to a strong damping of sound. In figure 9 , we show the axial velocity component $v_{z}(\boldsymbol{r}, t)$ as a function of $z / b$ and $R / b$ at time $t=1$. A comparison with figure 1 shows the influence of the increase in viscosity on the velocity field. Therefore, as in figure 1, only the velocity field for values of $R / b>0.2$ is shown. It is evident from the comparison that the viscosity has a very strong damping effect. In order to see the development in time towards $t=1$, in figures 10 and 11 we show the flow field at $t=0.4$ and $t=0.7$, respectively. In figure 12 , we show the radial velocity component $v_{R}(\boldsymbol{r}, t)$ as a function of $z / b$ and $R / b$ at time $t=0.7$. In figure 13, we show the streamlines at time $t=0.7$ in the plane $\varphi=0$. Again, no subsidiary vortices are seen beyond three diameters upstream or downstream of the point source in the range of time considered. A comparison between figures 1 and 9 indicates that the decrease of the amplitude of the vortex is about a factor 10 larger in this case. It takes a certain time for this vortex ring to develop, but at $t=2$ for the case $R_{b}=100$ and at $t=0.7$ for the case $R_{b}=5.3$, the vortex already exists, as 


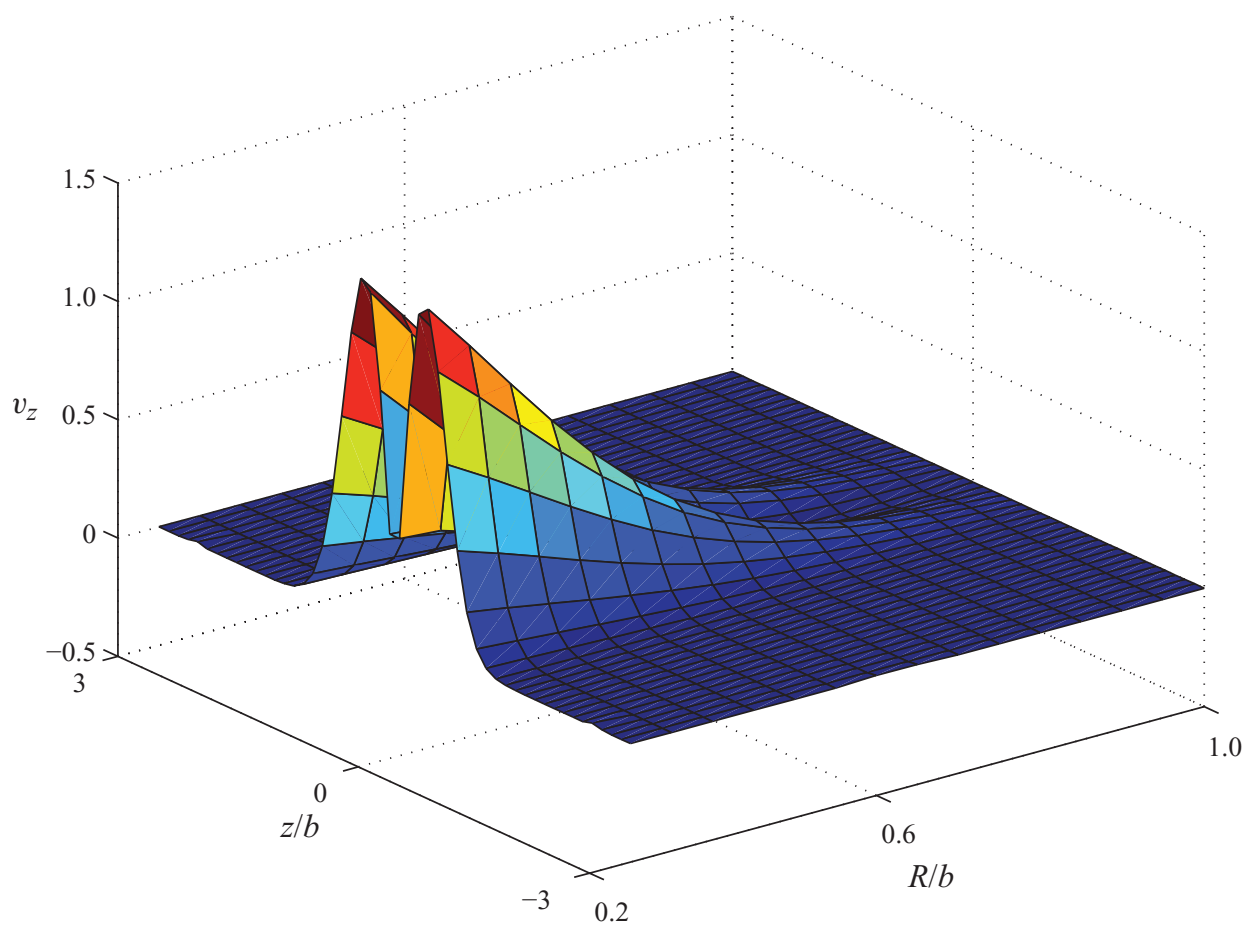

FIGURE 10. Plot of the axial velocity $v_{z}$ as a function of the axial coordinate $z / b$ and the radial coordinate $R / b$ at time $t=0.4$ and for $\eta=0.133$ and $\eta_{v}=0.00044$.

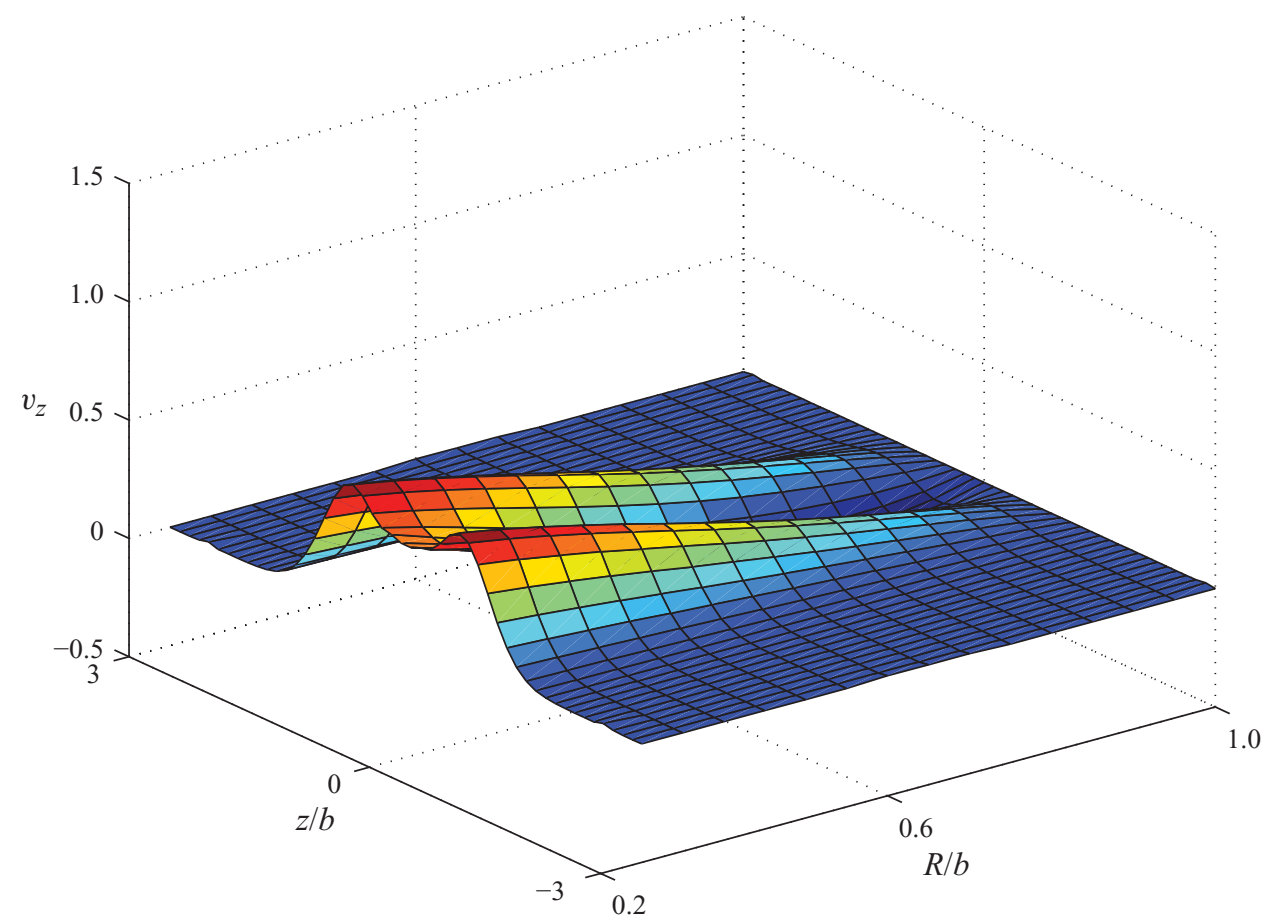

FIGURE 11. Plot of the axial velocity $v_{z}$ as a function of the axial coordinate $z / b$ and the radial coordinate $R / b$ at time $t=0.7$ and for $\eta=0.133$ and $\eta_{v}=0.00044$. 


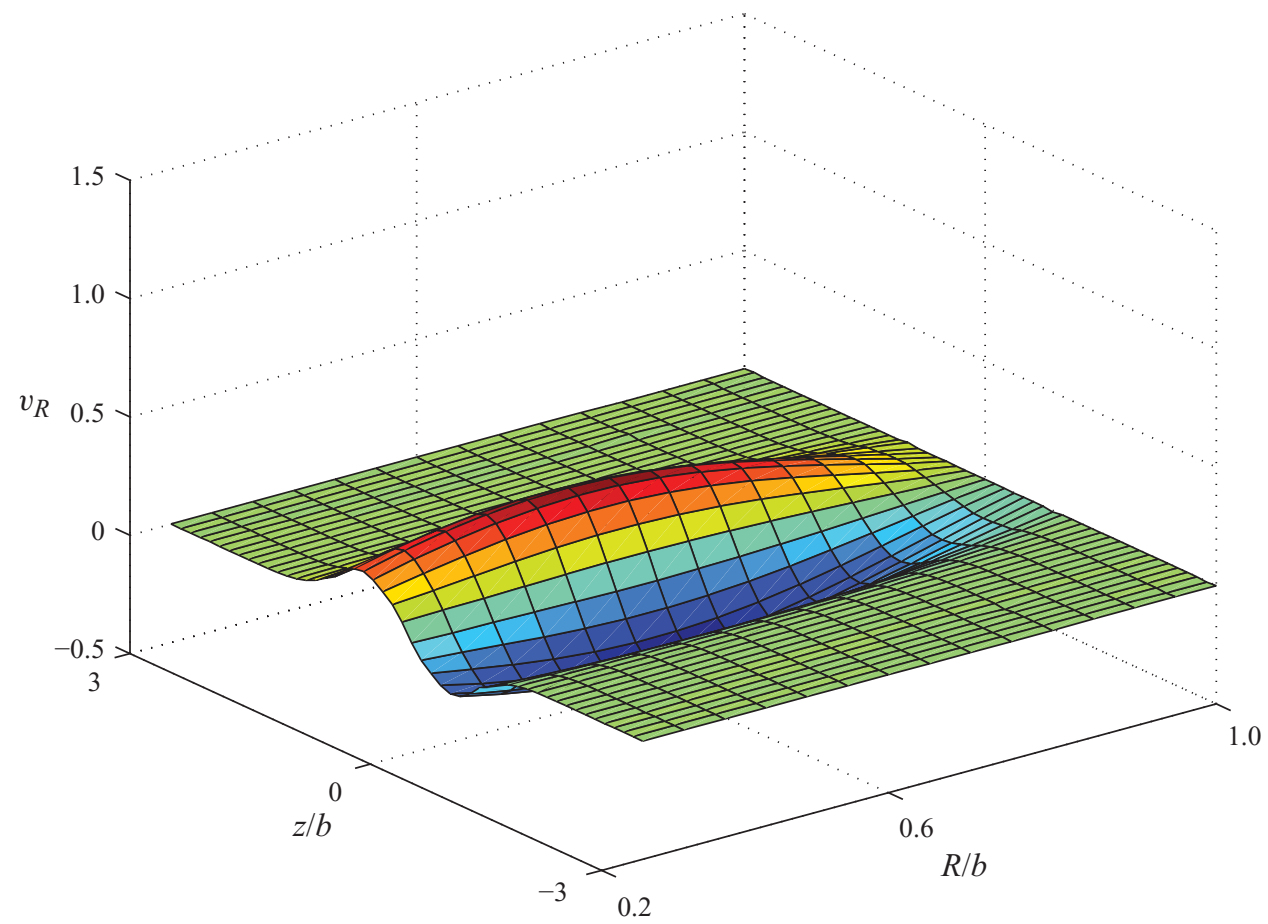

FIgURE 12. Plot of the radial velocity $v_{R}$ as a function of the axial coordinate $z / b$ and the radial coordinate $R / b$ at time $t=0.7$ and for $\eta=0.133$ and $\eta_{v}=0.00044$.

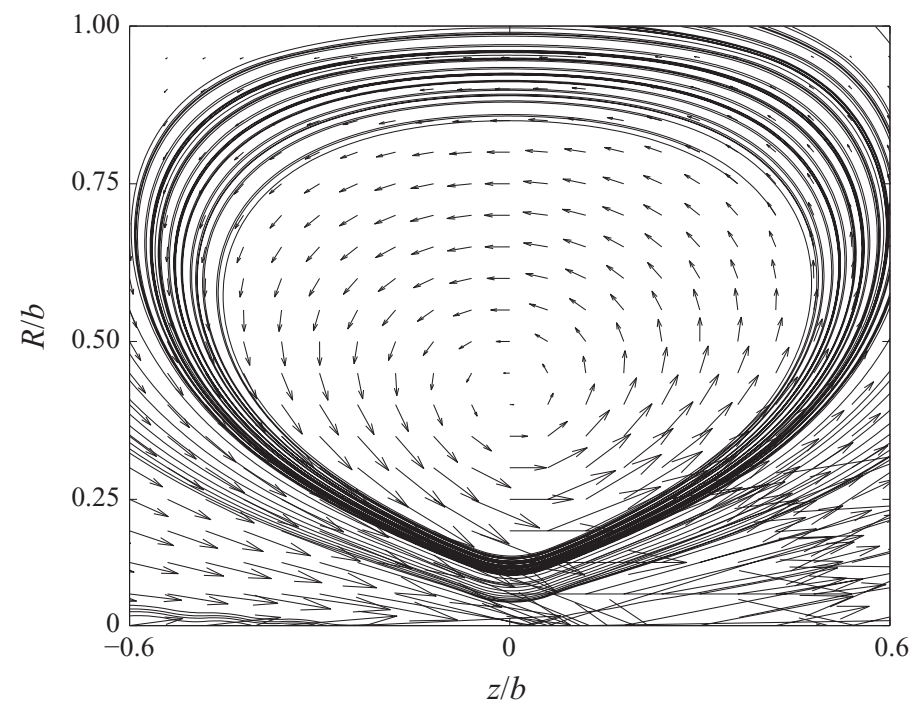

FIGURE 13. Plot of the velocity vectors and streamlines in the vortex ring at $t=0.7$ and for $\eta=0.133$ and $\eta_{v}=0.00044$.

shown in figures 5 and 13. In figure 14, we show the axial velocity component in the plane $z=0$ at time $t=0.7$ as a function of the radial distance $R / b$. For larger values of $|z / b|$, the flow is dominated by pressure waves. In figure 15 , we show the pressure disturbance $\delta p(\boldsymbol{r}, t)$ as a function of $R / b$ and $z / b$ at time $t=0.7$. In figure 16 , we show 


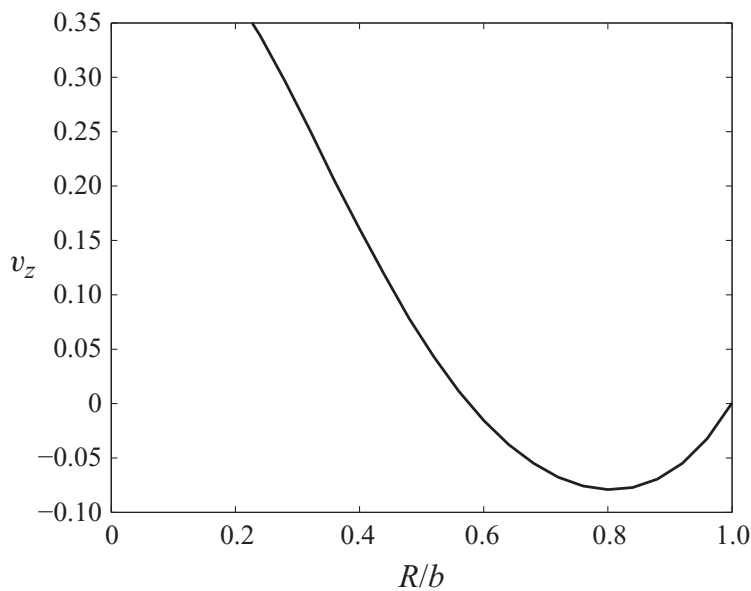

FIGURE 14. Plot of the axial velocity $v_{z}$ as a function of the radial coordinate $R / b$ at $z / b=0$ and $t=0.7$ and for $\eta=0.133$ and $\eta_{v}=0.00044$.

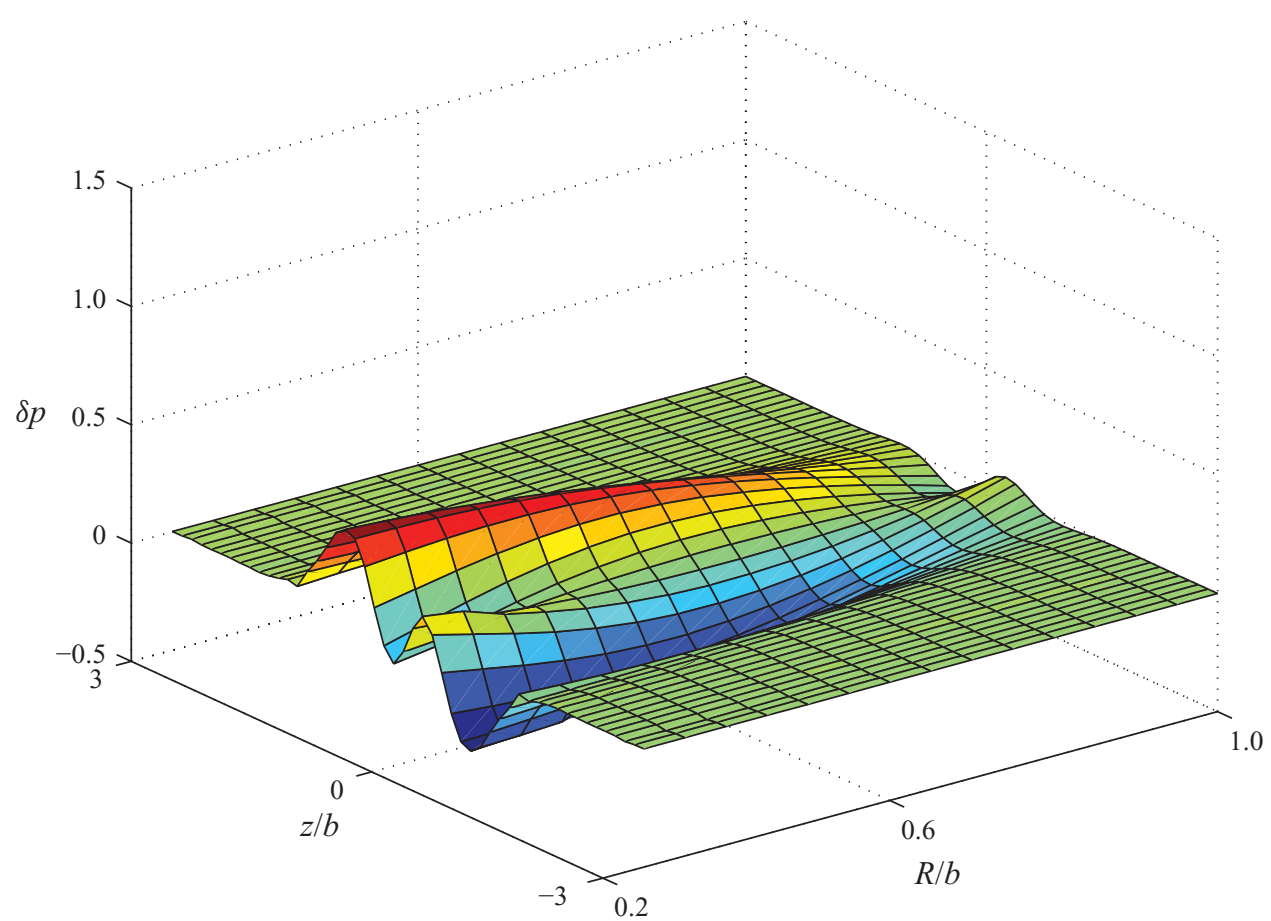

Figure 15. Plot of the pressure $\delta p$ as a function of the axial coordinate $z / b$ and the radial coordinate $R / b$ at time $t=0.7$ and for $\eta=0.133$ and $\eta_{v}=0.00044$.

the axial velocity component $v_{z}$ at the selected point $R / b=0.7, \varphi=0$ and $z / b=1$ as a function of time, as well as the separate contributions from the outgoing wave $v_{0 z}$ and the reflected wave $v_{1 z}$. The wave peaks in figure 16 are considerably broader than those in figure 8 . This is due to the dispersive effect of the viscosity, which is stronger for $R / b=5.3$ than for $R / b=100$. 


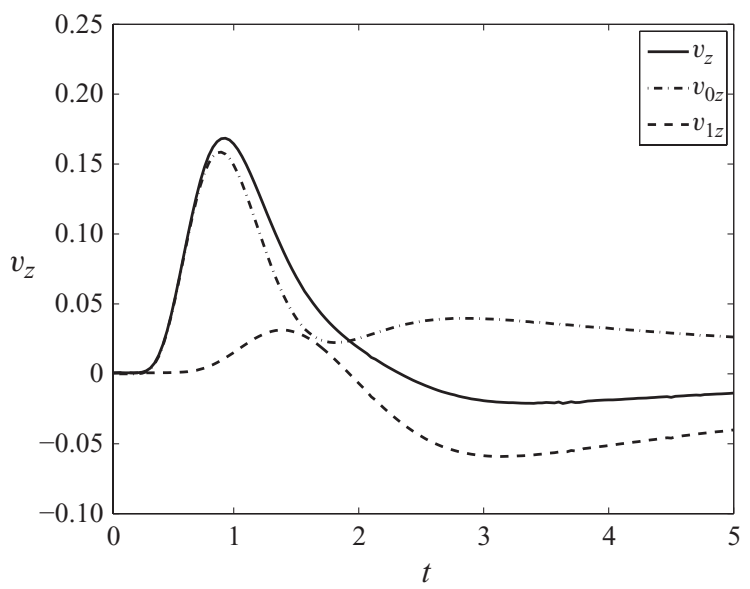

Figure 16. Plot the axial velocity $v_{z}$ at the selected point $(z / b=1, R / b=0.7)$ as a function of time $t$, as well as the separate contributions from the outgoing wave $v_{0 z}$ and the reflected wave $v_{1 z}$ for $\eta=0.133$ and $\eta_{v}=0.00044$.

\section{Discussion}

We have developed a technique for the efficient calculation of flow velocity and pressure as functions of position and time in a circular tube after a sudden impulse has been applied to the fluid on the axis of the tube. This allows a more complete picture of the flow behaviour than that previously obtained. The detailed results allow comparison with those obtained in computer simulation, permitting validation of the latter. It may be expected that the same technique can be applied to similar problems.

\section{REFERENCES}

Brigham, E. O. 1974 The Fast Fourier Transform. Prentice-Hall.

Cichocki, B. \& Felderhof, B. U. 2000 Long-time tails in the solid-body motion of a sphere immersed in a suspension. Phys. Rev. E 62, 5383-5388.

FelderhoF, B. U. 2010a Transient flow of a viscous compressible fluid in a circular tube after a sudden point impulse. J. Fluid Mech. 644, 97-106.

FeLDERHOF, B. U. $2010 b$ Transient flow of a viscous compressible fluid in a circular tube after a sudden point impulse transverse to the axis. J. Fluid Mech. 649, 329-340.

Frydel, D. \& Diamant, H. 2010 Long-range dynamic correlations in confined suspensions. Phys. Rev. Lett. 104, 248302.

Hagen, M. H. J., Pagonabarraga, I., Lowe, C. P. \& Frenkel, D. 1997 Algebraic decay of velocity fluctuations in a confined fluid. Phys. Rev. Lett. 78, 3785-3788.

JONES, R. B. 1981 Hydrodynamic fluctuation forces. Physica A 105, 395-416.

Liron, N. \& Sharar, R. 1978 Stokes flow due to a Skokeslet in a pipe. J. Fluid Mech. 86, 727-744.

Pagonabarraga, I., Hagen, M. H. J., Lowe, C. P. \& Frenkel, D. 1999 Short-time dynamics of colloidal suspensions in confined geometries. Phys. Rev. E 59, 4458-4469. 